\title{
Dieta de cinco espécies de Hemiodontidae (Teleostei, Characiformes) na área de influência do reservatório de Balbina, rio Uatumã, Amazonas, Brasil
}

\author{
Cylene C. da Silva, Efrem J. G. Ferreira \& Cláudia P. de Deus
}

Coordenação de Pesquisas em Biologia Aquática - CPBA, Instituto Nacional de Pesquisas da Amazônia - INPA. Caixa Postal 478, 69011970 Manaus, AM. (cylenecamara@yahoo.com.br; efrem@inpa.gov.br; claudias@inpa.gov.br).

\begin{abstract}
Diet of five species of Hemiodontidae (Teleostei, Characiformes) in the area of influence of the Balbina reservoir, Uatumã River, State of Amazonas, Brazil. The diet of five species of Hemiodontidae (Hemiodus argenteus (Pellegrin, 1908), H. atranalis (Fowler, 1940), H. immaculatus (Kner, 1858), H. microlepis (Kner, 1858) e H. unimaculatus (Bloch, 1794)) from the area of influence of the Balbina Reservoir, Uatumã River, northern Brazil, was investigated. Fish were collected bimonthly from April 2005 to February 2007 in four places, two in the reservoir (upstream the dam) and two in the river (downstream the dam). A total of 318 stomachs were analyzed. The diet was evaluated using two methods: frequency of occurrence and relative volume, both combined in the Alimentary Index (IAi). The breadth of the alimentary niche was low among the five analyzed species. Hemiodus unimaculatus presented the largest niche breadth, while $H$. argenteus showed the smallest. Detritus was an important item in the diet of all species, being predominant in Hemiodus argenteus, $H$. microlepis and $H$. unimaculatus. Hemiodus atranalis ingested mainly microcrustaceans and $H$. immaculatus consumed a larger proportion of vegetables. Despite the consumption of certain preferential items the ingestion of a wide variety of items occurs for all the species showing a broad trophic diversity in the diet of this family, which could explain its relative success in reservoirs in the Amazon.
\end{abstract}

KEYWORDS. Hemiodus, environmental impact, food habit, Amazon fish.

RESUMO. A dieta de cinco espécies de Hemiodontidae (Hemiodus argenteus (Pellegrin, 1908), H. atranalis (Fowler, 1940), H. immaculatus (Kner, 1858), H. microlepis (Kner, 1858) e H. unimaculatus (Bloch, 1794)) na área de influência do reservatório de Balbina, rio Uatumã, norte do Brasil, foi investigada. Os peixes foram coletados bimestralmente entre abril de 2005 e fevereiro de 2007 , em quatro locais, dois no reservatório (a montante da barragem) e dois no rio (a jusante da barragem). A dieta foi avaliada usando-se dois métodos: freqüência de ocorrência e volume relativo, combinados no Índice Alimentar (IAi). A amplitude do nicho alimentar foi baixa entre as cinco espécies analisadas. Hemiodus unimaculatus apresentou a maior amplitude de nicho, enquanto $H$. argenteus apresentou o menor. Detrito foi um item importante na dieta de todas as espécies analisadas, sendo predominante em H. argenteus e H. microlepis. Para $H$. unimaculatus, além dos detritos, as algas filamentosas também tiveram grande participação na alimentação da espécie. Já em $H$. atranalis e H. immaculatus, o item detrito também teve relativa importância na dieta, porém os itens microcrustáceos e vegetais foram, respectivamente, mais abundantes. Apesar da ampla variedade trófica, ocorreu o consumo preferencial de determinados itens, representados, principalmente, por detritos, matéria vegetal e microcrustáceos. A ampla diversidade trófica pode explicar o relativo sucesso das espécies desta família em ambientes represados.

PALAVRAS-CHAVE. Hemiodus, impacto ambiental, hábito alimentar, peixes da Amazônia.

O estudo da dieta de peixes e da interação alimentar destes com o meio fornece importantes informações ecológicas, bem como sobre o comportamento destas espécies diante de variações nas condições ambientais e na disponibilidade do alimento. Essas informações trazem subsídios para a compreensão dos mecanismos que permitem a coexistência e a exploração dos recursos por várias espécies em um mesmo sistema (GouLDING, 1980).

A disponibilidade de alimentos é um dos principais fatores para o sucesso de peixes em reservatórios (PAIVA, 1983; PETTS, 1984), de modo que é fundamental conhecer seus hábitos alimentares a fim de avaliar os impactos do represamento sobre o comportamento alimentar da ictiofauna. Algumas espécies encontram, no novo ambiente, condições favoráveis à proliferação, enquanto outras tendem à extinção local (HAHN et al., 1998).

Muitos autores, analisando o efeito das barragens na estrutura trófica da comunidade, verificaram a ocorrência de alterações marcantes na dieta das espécies após o represamento, com uma diversificação ou modificação dos itens alimentares ingeridos pela maior parte delas (Mérona et al., 2001; CASSEMiro et al., 2005).
Segundo HaHn et al. (1998), o estabelecimento de novas condições ambientais e as flutuações na disponibilidade de recursos decorrentes do represamento, devem favorecer as espécies dotadas de plasticidade alimentar, sendo assim, capazes de melhor se adaptarem ao novo ambiente.

As espécies pertencentes à família Hemiodontidae parecem apresentar relativo sucesso em ambientes represados na Amazônia. Ferreira (1984) cita Hemiodopsis sp. (=Hemiodus argenteus) como sendo uma das mais abundantes no reservatório de Curuá-Una. Em Tucuruí, Anodus orinocensis (Steindachner, 1887), Hemiodus unimaculatus (Bloch, 1794) e H. microlepis (Kner, 1858) estão entre as espécies de peixes mais exploradas e abundantes no reservatório (SANTOS et al., 2004). Hemiodus unimaculatus também está entre as espécies com maior freqüência de ocorrência no lago da Hidrelétrica de Samuel (RO), representando 26\% das capturas, e a família Hemiodontidae também é abundante na Hidrelétrica do Pitinga, localizada nas cabeceiras do rio Uatumã (AM) (SANTOS, 1995).

Após 10 anos do fechamento do reservatório de Samuel, SANTos (1995) constatou que os hemiodontídeos 
haviam se adaptado às novas condições e se estabelecido com maior sucesso no novo ambiente. $\mathrm{O}$ autor cita que a alta taxa de decomposição de troncos da floresta alagada, somada à maior penetração de luz e conseqüente aumento da camada eufótica, influenciou o aumento na abundância de organismos planctônicos e perizoo, os quais se constituem nas principais fontes alimentares deste grupo de peixes. Na área de influência da UHE Balbina, as espécies da família Hemiodontidae estão presentes em todos os ambientes (Efrem Ferreira, dados não publicados).

Neste estudo foi investigada a dieta de cinco espécies de Hemiodontidae [Hemiodus argenteus (Pellegrin, 1908); H. atranalis (Fowler, 1940); H. immaculatus (Kner, 1858); H. microlepis e $H$. unimaculatus] com o objetivo de verificar diferenças interespecíficas na dieta, bem como determinar os principais recursos alimentares utilizados por estas espécies na área de influência do reservatório de Balbina.

\section{MATERIAL E MÉTODOS}

O estudo foi realizado na área de influência da Hidrelétrica de Balbina, situada no rio Uatumã, município de Presidente Figueiredo, Amazonas, a cerca de 180 km de Manaus. A bacia hidrográfica do rio Uatumã localizase entre os paralelos $1^{\circ}$ e $3^{\circ} \mathrm{Sul}$ e os meridianos $58^{\circ}$ e $61^{\circ}$ Oeste, entre os estados do Amazonas e Roraima, totalizando uma área de drenagem de $70.600 \mathrm{~km}^{2}$ (RADAMBRASIL, 1976). O perfil do rio Uatumã é de baixa declividade na maior parte de sua extensão, exceto nas zonas de corredeiras e cachoeiras, das quais Morena e Balbina eram as mais importantes, sendo esta última o local de instalação da Hidrelétrica Balbina. Acima da cachoeira Balbina o leito era bem delimitado e as áreas inundadas pelas enchentes naturais do rio eram estreitas, sendo fortemente influenciadas pelas chuvas locais (SANTOS \& JEGU, 1996). A cachoeira Balbina deixou de existir após o fechamento do reservatório em 1987.

O reservatório formado desde 1987 possui uma área de $2.360 \mathrm{~km}^{2}$ (FEARNSIDE, 1990). Na margem esquerda está localizada a Reserva Biológica do Uatumã, criada em 1990 com o intuito de proteger amostras representativas dos ecossistemas das bacias dos rios Uatumã e Jatapu. A reserva abrange cerca de $560 \mathrm{mil} \mathrm{ha,} \mathrm{compreendendo} \mathrm{os}$ municípios de Presidente Figueiredo e São Sebastião do Uatumã (IBAMA, 2006).

Os quatro pontos de coleta estão situados na área de influência da Hidrelétrica Balbina, sendo dois localizados na área a jusante da barragem (1 - Cachoeira Morena - 02 $07^{\prime} 23,6^{\prime \prime}$ S e 59 $9^{\circ} 19^{\prime} 49,3$ '”W e 2 - Jusante da Barragem - 01 ${ }^{\circ} 55^{\prime} 11,8^{\prime}$ 'S e $59^{\circ} 28^{\prime} 19,0$ '' W) e dois localizados a montante, na área do reservatório (3 Montante da Barragem - 01'54'23''S e 59²7'39,4' W e 4 - Base Waba - 01³1'19,5'S e 5949'18,0”W) (Fig. 1).

As coletas foram realizadas bimestralmente, de abril de 2005 a fevereiro de 2007, utilizando-se redes de espera com malhas variando de 12 a $60 \mathrm{~mm}$ entre nós opostos. As redes permaneceram armadas durante 8 horas, divididas em dois períodos, das 4 às 8 horas e das 16 às 20 horas. Ao final de cada período estas eram despescadas e retiradas.

Após as despescas, os peixes que ainda estavam vivos foram anestesiados com solução de benzocaina e colocados em sacos plásticos. Posteriormente, todos os peixes coletados foram identificados e dissecados para a retirada dos estômagos, que foram etiquetados e preservados em álcool $70 \%$ para análise do conteúdo estomacal. Exemplares testemunhos das espécies foram depositados na Coleção de Peixes do Instituto Nacional de Pesquisas da Amazônia (INPA 25540, INPA 25517, INPA 25509, INPA 25567 e INPA 26864).

A análise da dieta foi realizada utilizando-se o método de frequiência de ocorrência (HysLop, 1980) e o método volumétrico (método dos pontos proposto por Hynes (1950) e modificado por SoAREs (1979)). Após a abertura do estômago, determinou-se o grau de repleção $(0 \%, 10 \%, 25 \%, 50 \%, 75 \%$ e $100 \%)$ (adaptação do método de Goulding et al., 1988). A abundância relativa de cada item foi estimada visualmente, considerando-se o total de alimento presente no estômago como sendo $100 \%$. Os valores estimados foram multiplicados pelo grau de repleção do estômago para correção do volume relativo. Os métodos freqüência de ocorrência e volumétrico foram combinados no Índice Alimentar (IAi) (KAWAKAMI \& VAZZOLER, 1980), cujos valores variam entre 0 e 1 ( $1 \geq \mathrm{IAi} \geq 0)$, como descrito pela equação abaixo:

$$
I A i=F i V i / \sum_{i=1}^{n}(F i V i)
$$

Onde: $\mathrm{i}=$ item alimentar que varia de 1 a $\mathrm{n} ; \mathrm{Fi}=$ frequiência de ocorrência $(\%)$ do item $\mathrm{i} ; \mathrm{Vi}=$ volume $(\%)$ do item $i$.

Os valores do IAi foram posteriormente convertidos em percentagens e utilizados para determinar a categoria trófica da espécie. Itens que apresentaram IAi superior a $50 \%$ determinaram a categoria trófica da espécie. Quando um único item não atingia este valor, os itens de maiores valores foram somados para determinar a categoria trófica.

A amplitude do nicho ecológico de cada espécie foi determinada pelo índice de Levins padronizado $\left(\mathrm{B}_{\mathrm{A}}\right)$ (KREBS, 1989):

$$
B_{A}=B-1 / n-1
$$

Onde: $\mathrm{B}_{\mathrm{A}}=$ índice padronizado de Levins, $\mathrm{B}=$ medida de amplitude de nicho (estimado por $1 / \Sigma \mathrm{p}_{\mathrm{j}}^{2}$ ), $\mathrm{pj}=$ fração do item da categoria "j" na dieta e $n=$ número de recursos utilizados. $\mathrm{B}_{\mathrm{A}}$ varia de 0 a 1 , sendo que valores próximos a zero indicam nicho trófico mais estreito.

\section{RESULTADOS}

Foi capturado um total de 586 exemplares das cinco espécies de Hemiodus. Destes, apenas 318 apresentavam algum conteúdo gástrico, sendo utilizados na análise da dieta. A espécie mais abundante foi $H$. argenteus, seguida por $H$. atranalis, $H$. immaculatus, $H$. microlepis e $H$. unimaculatus (Tab. I).

Os itens alimentares encontrados nos estômagos foram agrupados em quinze categorias: fitoplâncton (Baccilariophyceae, Chlorophyceae, Cyanobacteria, Euglenophyceae, Zygnemataceae, Chrysophyceae); algas filamentosas (Spyrogira sp.); vegetais (fragmentos vegetais, sementes, flores, frutos e macrófitas aquáticas); detritos (material orgânico particulado, não sendo possível distinguir sua origem, se animal ou vegetal); rotíferos; microcrustáceos (copépodos e cladóceros); Ostracoda; Conchostraca (Cyclestheria hislopi); Arachnida (aranhas e ácaros); espículas de Porifera; insetos imaturos 


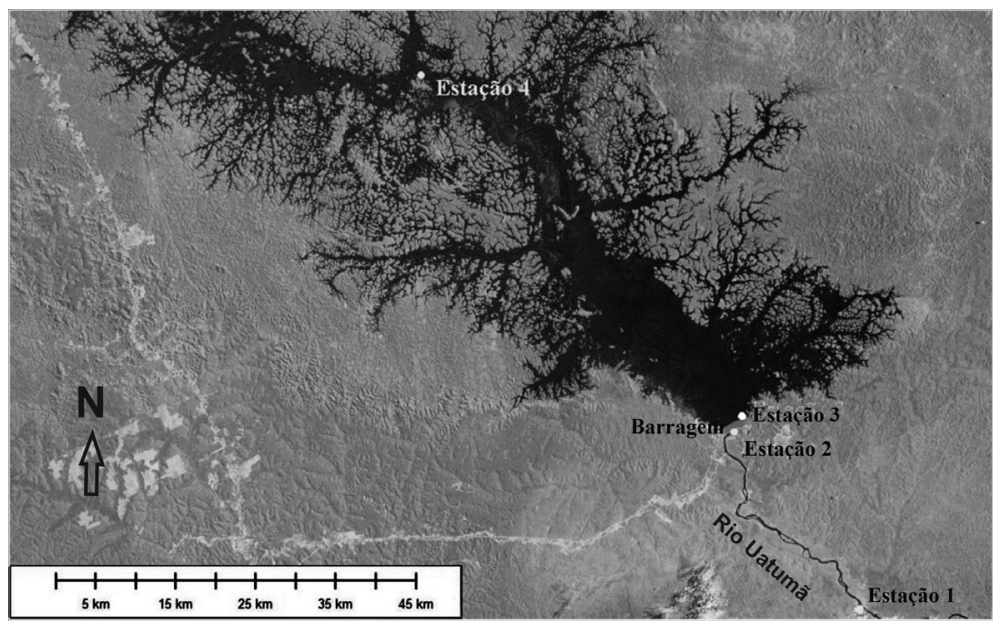

Fig. 1. Localização geográfica das quatro estações de coleta na área de influência do reservatório da UHE Balbina, Amazonas.

Tabela I. Índice Alimentar (IAi); número total de indivíduos capturados (N); número de indivíduos com conteúdo gástrico analisados (n); variação do comprimento padrão $(\mathrm{CP})$ e coeficientes de amplitude de nicho alimentar $\left(\mathrm{B}_{\mathrm{A}}\right.$ ) das espécies de Hemiodus analisadas durante o período de abril de 2005 a fevereiro de 2007, na área de influência do reservatório da UHE Balbina, Amazonas.

\begin{tabular}{|c|c|c|c|c|c|}
\hline \multicolumn{6}{|c|}{ ÍNDICE ALIMENTAR (IAi) } \\
\hline ESPÉCIES & H. argenteus & H. microlepis & H. atranalis & H. unimaculatus & H. immaculatus \\
\hline CATEGORIA TRÓFICA & Detritívoro & Detritívoro & $\begin{array}{c}\text { Zooplanctívoro/ } \\
\text { detritívoro }\end{array}$ & $\begin{array}{c}\text { Algívoro/ } \\
\text { detritívoro }\end{array}$ & $\begin{array}{l}\text { Herbívoro/ } \\
\text { detritívoro }\end{array}$ \\
\hline \multicolumn{6}{|l|}{ ITENS } \\
\hline Fitoplâncton & 0,420 & 7,028 & 0,301 & 6,623 & 4,290 \\
\hline Algas filamentosas & 1,190 & 1,723 & 1,002 & 30,728 & 3,677 \\
\hline Vegetais & 0,129 & 8,669 & 14,086 & 14,305 & 55,669 \\
\hline Detritos & 97,733 & 81,825 & 30,106 & 47,682 & 20,843 \\
\hline Rotifera & $<0,001$ & & & & \\
\hline Microscrustáceo & 0,010 & 0,110 & 45,904 & & 0,018 \\
\hline Ostracoda & 0,011 & 0,002 & 7,288 & & 0,186 \\
\hline Conchostraca & 0,317 & 0,234 & & & 0,017 \\
\hline Arachnida & 0,066 & & 0,063 & & \\
\hline Porifera & 0,004 & 0,013 & & 0,199 & \\
\hline Insetos imaturos & 0,131 & 0,043 & 1,175 & 0,199 & 12,851 \\
\hline Insetos aquáticos adultos & 0,003 & & & & 0,133 \\
\hline Insetos terrestres adultos & 0,015 & & 0,026 & & \\
\hline Frag. insetos não identif. & 0,001 & 0,406 & 0,041 & 0,265 & 2,295 \\
\hline Peixes & & 0,353 & 0,009 & & 0,022 \\
\hline $\mathrm{N}(\mathrm{n})$ & $237(183)$ & $45(27)$ & $203(63)$ & $29(16)$ & $72(29)$ \\
\hline CP (mín- máx) & 79-124 & $87-210$ & $65-101$ & $98-190$ & $117-190$ \\
\hline $\mathrm{B}_{\mathrm{A}}$ & 0,004 & 0,047 & 0,206 & 0,314 & 0,168 \\
\hline
\end{tabular}

(larva, pupa e ninfa de Ephemeroptera, Diptera, Trichoptera, Coleoptera, Lepidoptera, Hemiptera e Odonata); insetos aquáticos adultos (Coleoptera e Hemiptera); insetos terrestres adultos (Isoptera, Hymenoptera, Orthoptera, Hemiptera, Odonata, Coleoptera, Diptera, Homoptera e Lepidoptera); fragmentos de insetos não identificados e peixes (escamas, músculo, otólitos, raios de nadadeiras e peixes inteiros).

A dieta das espécies apresentou grande variedade de itens alimentares, porém, poucos foram predominantes. Detritos foi um item preponderante na dieta de todas as espécies, entretanto, apenas para $H$. argenteus e $H$. microlepis o item apresentou percentual superior a $50 \%$. Para Hemiodus unimaculatus, além dos detritos, as algas filamentosas também apresentaram significativa participação na dieta, enquanto que, para H. atranalis, os microcrustáceos foram mais abundantes, seguido pelo item detritos. Hemiodus immaculatus ingeriu principalmente vegetais e detritos (Tab. I).

Apenas Hemiodus argenteus e H. microlepis foram capturadas nas quatro estações de coleta e o consumo de detrito foi sempre predominante em todas elas. Hemiodus atranalis ocorreu nas estações 1, 3 e 4, onde consumiu, principalmente, detritos; microcrustáceos e detritos; e detritos, ostracoda e insetos imaturos, respectivamente. As espécies Hemiodus unimaculatus e H. immaculatus foram capturadas nas estações 1 e 2. Hemiodus unimaculatus consumiu, principalmente, detritos e algas filamentosas em ambas as estações, enquanto $H$. immaculatus consumiu vegetais e detritos na estação 1 e principalmente vegetais na estação 2 (Tab. II). Quanto à ocorrência das espécies ao longo do período de 
Tabela II. Índice Alimentar (IAi) de cinco espécies de Hemiodus nas estações de coleta, durante o período de abril de 2005 a fevereiro de 2007, na área de influência do reservatório da UHE Balbina, Amazonas.

\begin{tabular}{|c|c|c|c|c|c|c|c|c|c|c|c|c|c|c|}
\hline & & & & & & ÍNDIC & CE ALIM & MENTAR & $\mathrm{R}(\mathrm{IAi})$ & & & & & \\
\hline & & H. arg & genteus & & & H. $n$ & nicrolepis & & & H.atranal & & H. unimo & aculatus & H. immaculatus \\
\hline & & Esta & ç̧ões & & & & stações & & & Estações & & Estaç & ções & Estações \\
\hline ITENS & 1 & 2 & 3 & 4 & 1 & 2 & 3 & 4 & 1 & 3 & 4 & 1 & 2 & 2 \\
\hline Fitoplâncton & 0,100 & & 0,133 & 0,247 & 1,493 & & 24,269 & & 30,0 & 0,191 & & 12,544 & 0,847 & 2,198 \\
\hline $\begin{array}{l}\text { Algas } \\
\text { filamentosas }\end{array}$ & 15,152 & & 1,160 & 0,728 & 4,181 & & 1,044 & 0,783 & & 1,161 & & 25,261 & 36,864 & 4,455 \\
\hline Vegetais & & 1,107 & 0,133 & 0,139 & 46,341 & & 3,445 & 0,261 & & 19,207 & 0,361 & 21,603 & 4,237 & $74,60586,792$ \\
\hline Detritos & 84,485 & 98,893 & 98,440 & 97,964 & 47,666 & 100,0 & 71,242 & 94,017 & 50,0 & 26,651 & 28,606 & 40,070 & 55,085 & $22,4445,660$ \\
\hline Rotifera & & & & $>0,001$ & & & & & & & & & & \\
\hline Microscrustácec & & & 0,002 & 0,006 & 0,050 & & & 0,704 & 20,0 & 47,727 & 19,014 & & & 0,021 \\
\hline Ostracoda & & & 0,010 & 0,014 & 0,020 & & & & & 4,521 & 26,635 & & & $0,0057,547$ \\
\hline Conchostraca & & & 0,021 & 0,605 & & & & 2,365 & & & & & & 0,020 \\
\hline Arachnida & & & & & & & & & & 0,092 & & & & \\
\hline Porífera & 0,263 & & & 0,002 & 0,149 & & & & & & & 0,523 & & \\
\hline Insetos imaturos & & & & 0,286 & 0,050 & & & & & 0,041 & 25,385 & & 1,271 & 15,570 \\
\hline $\begin{array}{l}\text { Insetos aquático } \\
\text { adultos }\end{array}$ & & & 0,047 & & & & & & & & & & & 0,161 \\
\hline $\begin{array}{l}\text { Insetos terrestre } \\
\text { adultos }\end{array}$ & & & 0,041 & 0,009 & & & & & & 0,037 & & & & \\
\hline $\begin{array}{l}\text { Frag. insetos } \\
\text { não identif. }\end{array}$ & & & 0,014 & & & & & & & 0,059 & & & 1,695 & 2,493 \\
\hline Peixes & & & & & 0,050 & & & 1,739 & & 0,013 & & & & 0,027 \\
\hline $\mathrm{N}^{\mathrm{o}}$ exemplares & 13 & 4 & 44 & 122 & 9 & 1 & 10 & 7 & 2 & 50 & 11 & 9 & 7 & 27 \\
\hline
\end{tabular}

estudo, a maioria foi coletada em quase todos os meses de amostragem, apenas $H$. immaculatus foi coletado somente no mês de abril, que corresponde ao período de cheia.

De acordo com os resultados, $H$. argenteus e $H$. microlepis foram consideradas, neste estudo, como detritívoras, $H$. atranalis, como zooplanctívora/ detritívora, H. unimaculatus, como algívora/detritívora e H. immaculatus, como herbívora/detritívora.

A amplitude do nicho trófico $\left(\mathrm{B}_{\mathrm{A}}\right)$ das espécies analisadas pode ser considerada baixa, com valores entre 0,004 e 0,314. A espécie com maior $\mathrm{B}_{\mathrm{A}}$ foi $H$. unimaculatus, enquanto $H$. argenteus apresentou o menor valor (Tab. I).

\section{DISCUSSÃO}

Segundo Roberts (1974), os peixes da família Hemiodontidae habitam grandes lagos, rios e as florestas inundadas. São peixes longos, fusiformes, pelágicos e bons nadadores (SANTOS et al., 1984). Sua alimentação é constituída, principalmente, por restos de plantas, detritos e larvas de insetos (Goulding, 1980). De forma geral, esta última afirmativa corrobora os dados obtidos para os Hemiodontidae aqui estudados.

A alimentação de espécies de peixes do rio Uatumã foi avaliada na década de 1980, antes da construção da barragem de Balbina (Rosseval Leite, com. pess.). Da família Hemiodontidae, apenas $H$. unimaculatus havia sido coletada, sendo que as frequiências de ocorrência dos itens ingeridos registrados naquele estudo e no presente trabalho não apresentam diferenças significativas (Wilcoxon $\mathrm{p}=0,2$ ). Aparentemente, a construção da barragem não ocasionou nenhuma mudança na dieta dessa espécie, porém $H$. unimaculatus e H. immaculatus parecem estar atualmente restritas à região a jusante da barragem, onde as características lóticas predominam. Claro Jr. et al. (2004), estudando a espécie $H$. argenteus em lagos de várzea na Amazônia Central, observaram que a mesma apresentou hábito alimentar herbívoro e invertívoro. MÉRONA et al. (2001) analisou a dieta desta espécie antes e após o fechamento da barragem de Tucuruí, no rio Tocantins, Estado do Pará, classificando-a como detritívora antes do fechamento e como herbívora após o fechamento. Entretanto, os autores fazem à ressalva de que a espécie é provavelmente onívora, mudando a sua alimentação de acordo com os recursos disponíveis. Outros autores confirmam o hábito alimentar onívoro para as espécies desta família (FERREIRA et al., 1998; SANTOS et al., 2004). É provável que, em Balbina, seja grande o acúmulo de detrito em todas as áreas onde foram realizadas as coletas e $H$. argenteus permaneceu alimentando-se de detrito antes e após o fechamento. Esta grande disponibilidade de detritos no ambiente também pode ser corroborada pela presença significativa do item no conteúdo estomacal de todas as espécies analisadas.

Hemiodus immaculatus apresentou dieta herbívora/detritívora, alimentando-se principalmente de vegetais e detritos. HoEINGHAUs et al. (2003), analisando $H$. immaculatus, $H$. argenteus e $H$. unimaculatus em um tributário do rio Orinoco, classificaram as espécies como algívoras/detritívoras, mesmo hábito alimentar encontrado neste estudo para $H$. unimaculatus.

Com base nos resultados do índice alimentar (IAi) Hemiodus atranalis foi classificada como uma espécie zooplanctívora/detritívora, alimentando-se principalmente de microcrustáceos (cladóceros e copépodos) e detritos. A espécie só foi capturada na área a montante da barragem (estação 3 e 4), onde os ambientes com características lênticas na região litorânea favorecem o desenvolvimento da comunidade zooplanctônica (EsTEVES, 1988). Este é o primeiro registro na literatura científica da dieta desta espécie.

Numa comparação mais ampla entre os dados gerados neste trabalho e aqueles apresentados na literatura (FERREIRA et al., 1998; MÉRONA et al., 2001; CLARO JR. et al., 2004; SANTOS et al., 2004), é possível observar o caráter oportunista dessas espécies com relação a sua 
estratégia alimentar, pelo fato de utilizarem os recursos alimentares que presumivelmente estejam disponíveis no ambiente. Apesar de não terem sido feitas análises da oferta alimentar, WINEMILLER \& KELSO-WINEMILLER (1996) demonstraram que o conteúdo alimentar de diferentes espécies presentes num mesmo local poderia ser utilizado como uma medida indireta da disponibilidade de alimento no ambiente.

O caráter oportunista na dieta de peixes é relatado por Lowe-McConNELl (1999) para a maioria das espécies da bacia amazônica, embora a autora cite a possibilidade de haver algum grau de preferência, como observado neste estudo. Os resultados aqui obtidos demonstram certa plasticidade trófica das espécies estudadas, indicando prováveis ajustes em seus comportamentos alimentares frente às modificações ambientais, o que poderia explicar o relativo sucesso destas espécies em ambientes represados. Ainda que estes ajustes possam ocorrer, a amplitude de nicho não foi tão grande para nenhuma das espécies estudadas e, por isso, poderiam ser consideradas espécies oligofágicas, ou seja, que se alimentam de alguns poucos itens alimentares (BEGON et al., 1996). A preferência alimentar de espécies oligofágicas é determinada dentro de um leque de opções relativamente restrito e dentro do qual certo grau de preferência é sempre aparente.

A maioria das espécies foi registrada em todos os meses de coleta, exceto $H$. immaculatus, que apareceu somente em abril (período de cheia). A dinâmica do ciclo hidrológico em regiões sob influência de uma barragem é totalmente descaracterizada, visto que a variação no nível de água passa a ser feita, de forma artificial, por técnicos que mantém a vazão do rio em níveis estipulados para garantir a geração de energia (JunK \& NuNES DE MELlo, 1987; FEARNSIDE, 1990). Uma vez que as variações do nível da água do ciclo hidrológico natural não ocorrem na área do reservatório, estas não afetam a distribuição temporal das espécies. Entretanto, pode se dizer que as espécies encontradas ao longo de todo o rio atualmente restringem sua distribuição a locais onde características de ambientes lóticos são constantes (SANTOS \& JEGU, 1996).

Os dados obtidos neste trabalho contribuem para o entendimento da interação consumidor-ambiente e das adaptações de cada espécie frente às alterações causadas pela construção de uma barragem.

Agradecimentos. Os autores agradecem ao INPA (Instituto Nacional de Pesquisas da Amazônia) pela infra-estrutura, à FAPEAM (Fundação de Amparo à Pesquisa do Amazonas), ao CNPq, e à Manaus Energia S.A. pelo apoio financeiro e logístico e ao IBAMA pelas Permissões de Coleta (07/2005 e 07/2006).

\section{REFERÊNCIAS BIBLIOGRÁFICAS}

Begon, M.; Harper, J. L. \& Townsend, C. R. 1996. Ecology. Oxford, Blackwell Science. 1068p.

Cassemiro, F. A. S.; Hahn, N. S. \& Delariva, R. L. 2005. Estrutura trófica da ictiofauna, ao longo do gradiente longitudinal do reservatório de Salto Caxias (rio Iguaçu, Paraná, Brasil), no terceiro ano após o represamento. Acta Scientiarum 27:63-71.

Claro Jr., L. H.; Ferreira, E. J.G.; Zuanon, J. S. \& Araújo-Lima, C. A. R. M. 2004. O efeito da floresta alagada na alimentação de três espécies de peixes onívoros em lagos de várzea da Amazônia Central Brasil. Acta Amazonica 34:133-137.

Esteves, F. A. 1988. Fundamentos de Limnologia. Rio de Janeiro, Interciência. 575p

Fearnside, P. 1990. A hidrelétrica de Balbina: O faraonismo irreversível versus ambiente na Amazônia. São Paulo, Instituto de Antropologia e Meio Ambiente. 46p.

Ferreira, E. J. G. 1984. A ictiofauna da represa hidrelétrica de Curuá-Una. Santarém, Pará. II: Alimentação e hábitos alimentares das principais espécies. Amazoniana 9:1-16.

Ferreira, E. J. G.; Zunanon, J. A. S. \& Santos, G. M. 1998. Peixes comerciais do médio Amazonas: Região de Santarém, Pará. Brasília, IBAMA. 211p.

Goulding, M. 1980. The fishes and the forest. Exploration in Amazonian Natural History. Berkeley, University of California. 280p.

Goulding, M.; Carvalho, M. L. \& Ferreira, E. G. 1988. Rio Negro, rich life in poor water: Amazonian diversity and foodchain ecology as seen thought fish communites. California, SPB Academic. 200p.

Hahn, N. S.; Agostinho, A. A.; Gomes, L. C. \& Bini, L. M. 1998. Estrutura trófica da ictiofauna do reservatório de Itaipu (Paraná-Brasil) nos primeiros anos de sua formação. Interciência 23:299-305.

Hoeinghaus, D. J.; Layman, C. A.; Arrington, D. A. \& Winemiller, K. O. 2003. Spatiotemporal variation in fish assemblage structure in tropical floodplain creeks. Environmental Biology of Fishes 67:379-387.

Hynes, H. B. N. 1950. The food of freshwater sticklebacks (Gasterosteus aculeatus and Pygosteus pungitius) with a review of methods used in the studies of the food of fishes. Journal Animal Ecology 19:36-58

Hyslop, E. J. 1980. Stomach contents analysis a review of methods and their application. Journal Fish Biology 17:411-429.

iBAMA. Instituto Brasileiro do Meio Ambiente E dos Recursos Naturais Renováveis. 2006. Reserva Biológica do Uatumã Decreto 99.277. Disponível em: <http://www.ibama.gov.br/ siucweb/unidades/rebio/planosde_manejo/100/html/ index.htm>. Acesso em: 17.11.2006.

JunK, W. J. \& Nunes de Mello, J. A. S. 1987. Impactos ecológicos das represas hidrelétricas na bacia amazônica brasileira. Tübinger Geographische Studien 95:367-385.

Kawakami, E. \& Vazzoler, G. 1980. Método gráfico e estimativa de índice alimentar aplicado no estudo de alimentação de peixes. Boletim do Instituto Oceanografico 29:205-207.

KREBS, C. J. 1989. Ecological methodology. New York, Harper \& Row. 654p.

Lowe-McConnell, R. H. 1999. Estudos Ecológicos de comunidades de peixes tropicais. São Paulo, EDUSP. 534p.

Mérona, B.; Santos, G. M. \& Almeida, R. G. 2001. Short term effects of Tucuruí Dam (Amazonia, Brazil) on the trophic organization of fish communities. Environmental Biology of Fishes 60:375-392.

Paiva, M. P. 1983. Impacto das grandes represas sobre o meio ambiente. Revista Ciências e Cultura 35:1274-1282.

Petts, G. E. 1984. Impoundment river. Perspectives for ecological management. Chinchester, John Wiley \& Sons. 326p.

Radambrasil. 1976. Programa de integração nacional. Levantamento de recursos naturais. Rio de Janeiro, DNPM. v.10. 510p

Roberts, T. R. 1974. Osteology and classification of the Neotropical characoid fishes of the families Hemiodontidae (including Anostomidae) and Parodontidae. Bulletin of the Museum of Comparative Zoology 146:411-472.

Santos, G. M. 1995. Impactos da hidrelétrica Samuel sobre as comunidades de peixes do rio Jamari (Rondônia - Brasil). Acta Amazonica 25:247-280.

Santos, G. M. \& Jegu, M. 1996. Inventário taxonômico dos anostomídeos (Pisces, Anostomidae) da bacia do rio UatumãAM, Brasil, com descrição de duas espécies novas. Acta Amazonica 26:151-184.

Santos, G. M.; Jegu, M. \& Merona, B. 1984. Catálogo de peixes comerciais do baixo rio Tocantins: Projeto Tucuruí. Manaus, Eletronorte - INPA. 83p.

Santos, G. M.; Mérona, B.; Juras, A. A. \& Jégu, M. 2004. Peixes do baixo Tocantins: 20 anos depois da Usina Hidrelétrica de Tucuruí. Brasília, Eletronorte. 216p.

SoAres, M. G. M. 1979. Aspectos ecológicos (alimentação e reprodução) dos peixes do igarapé do Porto, Aripuanã, MT. Acta Amazonica 9:325-352.

Winemiller, K. O. \& Kelso-Winemiller, L. C. 1996. Comparative ecology of catfishes of the upper Zambesi river floodplain. Journal of Fish Biology 49:1043-1061.

Recebido em maio de 2007. Aceito em junho de 2008. ISSN 0073-4721

Artigo disponível em: www.scielo.br/isz 